\title{
La importancia de la infraestructura física en el crecimiento económico de los municipios de la frontera norte
}

\author{
Héctor Alonso Barajas Bustillos, ${ }^{*}$ Luis Gutiérrez Flores**
}

\section{Resumen}

El presente documento evalúa la importancia que tiene la infraestructura física económica en el crecimiento de los municipios de la frontera norte. Dentro de la literatura del crecimiento, la infraestructura, además de otros factores, ha sido señalada como un elemento determinante en las perspectivas de largo plazo al respecto del ritmo al cual crecen las regiones. Sin embargo, en este contexto temático aún son escasas las aportaciones que remiten a estudios en donde la unidad de análisis se desagrega hasta el nivel del municipio, que en el caso de la frontera norte de México constituye un escenario económico de mucha relevancia dado el modelo de desarrollo adoptado por la economía nacional en las últimas décadas. Los resultados de los modelos empíricos nos indican que los municipios fronterizos de Piedras Negras, Nogales y Torreón mantienen una relación positiva entre su crecimiento y la dotación física de infraestructura. En el caso de otros municipios como Tijuana, su elevado crecimiento poblacional inhibe la adecuada provisión de infraestructura, aunque se mantiene el efecto positivo que la infraestructura induce sobre el crecimiento.

Palabras clave: infraestructura, crecimiento económico, municipios, frontera norte, especialización industrial.

\section{Abstract}

This paper evaluates the importance of infrastructure on economic growth for the Mexican northern border municipalities. From the growth literature, we know that infrastructure, besides other factors, has been pointed out as a key factor in the long run perspectives of regional growth. Nevertheless, and within this thematic context, works that use a disaggregated analysis down to the level of the municipality are still scarce, which in the case of the northern border constitutes a scenario of much relevance given the development model adopted by the Mexican economy in recent decades. Empirical models results indicate that the municipalities of Piedras Negras, Nogales and Torreon maintain a positive relationship between physical infrastructure and growth. In the case of other municipalities like Tijuana, its elevated population growth inhibits a proper infrastructure allocation, although the positive effect induced by infrastructure on growth remains.

Keywords: infrastructure, economic growth, municipality, northern border, industrial specialization.

* Egresado del programa de Maestría en Economía Regional del Centro de Investigaciones Socioeconómicas de la Universidad Autónoma de Coahuila. Correo electrónico: hector.barajas@gmail.com.

** Profesor y director del Centro de Investigaciones Socioeconómicas de la Universidad Autónoma de Coahuila. Correo electrónico: luis.gutierrez@uadec.edu.mx. 


\section{Introducción}

El crecimiento en México es un tema importante ya que existen divergencias económicas regionales, esto es, diferencias marcadas entre el crecimiento y la productividad en los estados y las regiones (Fuentes, 2007). Para disminuir estas desigualdades se debe definir cuáles son los elementos que permiten potenciar el crecimiento regional. Dichos elementos pueden dividirse en tres grupos: los recursos naturales, en cuya formación los individuos difícilmente pueden interferir; los recursos humanos (oferta de trabajo, capacitación, disciplina, motivación, etcétera) y la formación de capital (maquinaria, fábricas, instalaciones, etcétera), los cuales son llevados a cabo generalmente por las mismas empresas; y finalmente, los elementos brindados por los gobiernos, como la educación, la seguridad pública, las legislaciones y la infraestructura. Este último es un elemento clave en los procesos de producción y en la mejora en la calidad de vida de los individuos, por lo que es necesario abundar en los estudios de su efecto en el crecimiento y desarrollo (Aschauer, 1990).

El documento se encuentra estructurado de la siguiente forma: en la primera sección se presenta una breve revisión teórica acerca de la relación entre el crecimiento y la convergencia; la segunda sección resalta el papel de la infraestructura en el análisis económico; en la tercera sección se desarrolla la estrategia empírica del documento al tiempo que se expone la evidencia que ha resultado de otros trabajos sobre el tema; finalmente, en la cuarta sección se presentan algunas conclusiones del trabajo.

Nuestros resultados apuntan hacia la confirmación de que la infraestructura es importante en el crecimiento económico de los municipios de la frontera norte de México, particularmente en Piedras Negras, Nogales y Torreón. Esta dinámica no escapa a grandes espacios económicos como Tijuana, sin embargo, algunos indicadores demográficos -como el crecimiento de la población- limitan la provisión eficiente de infraestructura.

\section{Crecimiento y convergencia}

A pesar de tener un origen macroeconómico, el crecimiento económico regional se caracteriza por la incorporación de características territoriales, donde la movilidad de factores modifica el desarrollo teórico propio de la 
macroeconomía (Capello, 2006). Esto es importante porque "la comprensión de cómo y por qué crece una región es esencial para la elaboración de una política regional eficaz" (Richardson, 1986).

En este sentido, a lo largo del tiempo los economistas han tratado de explicar las desigualdades entre países o regiones y le han dado cada vez mayor importancia a las teorías que explican cómo hacer crecer una economía. Así, dentro de la literatura del crecimiento económico se distinguen cinco grandes escuelas de pensamiento, a saber: los clásicos, los keynesianos, los neoclásicos, la nueva geografía económica y las nuevas teorías de crecimiento o crecimiento endógeno.

Los clásicos entendieron el crecimiento en función de los recursos naturales, las actividades primarias, la división del trabajo y el comercio internacional (Smith, 1958; Ricardo, 1973; Malthus, 1986; Ohlin, 1971; Heckscher, 1991).

Por otra parte, el principal argumento keynesiano se basa en los ciclos económicos y en lograr que el periodo de auge sea más duradero. Las variables importantes de este modelo son la inversión y el ahorro. Es por medio del multiplicador y del acelerador de la inversión -así como haciendo más duraderos los periodos de auge alargando el equilibrio- como se logra un crecimiento económico. En este enfoque no se manifiesta una preocupación explícita relacionada con disminuir las diferencias entre los niveles de crecimiento de los países ya que sólo se trata de un equilibrio local. Destaca la importancia de la intervención del gobierno en la economía (Keynes, 1992; Kaldor, 1963; Robinson, 1973 y 1974; Harrod, 1939; Domar, 1946; Hicks, 1963).

La escuela neoclásica explica el proceso de crecimiento mediante la formalización de un modelo matemático. Sin embargo, debido a los supuestos utilizados deja al factor de crecimiento -la tecnología- como un elemento exógeno que el modelo mismo no logra explicar (Solow, 1956; Sala-i-Martin, 1996).

En la nueva geografía económica se explica cómo afectan los costos de transporte a la localización de las empresas e individuos y cómo esas decisiones generan aglomeraciones y competencia monopolística que influyen en las habilidades de los individuos y las capacidades de las empresas (Fujita y Krugman, 2004).

Finalmente, el crecimiento endógeno abandona algunos de los supuestos neoclásicos para explicar el crecimiento con factores como la educación, 
la innovación, las ideas y el gasto del gobierno, entre otros elementos que influyen en el capital humano (Barro, 1990; Romer, 1994).

En el modelo neoclásico de crecimiento, debido al supuesto de rendimientos decrecientes en los factores, el país crece más rápido cuando está por debajo de su estado estacionario, mientras que crecerá más lento cuando esté por encima de éste. A partir de esta dinámica se explican las diferencias entre las tasas de crecimiento de distintos países. A dicha dinámica se le conoce como proceso de convergencia (Sala-i-Martin, 1995). En las teorías de crecimiento endógeno este supuesto se elimina, por lo que los países pueden tener tasas de crecimiento constantes sin llegar a la convergencia, o bien tasas crecientes llevando al afecto contrario llamado divergencia. En el crecimiento económico regional, la movilidad de los factores desempeña un papel importante en estos procesos.

La teoría de la convergencia ha sido abordada en México por diversos investigadores (Calderón y Tykhonenko, 2006; Esquivel, 2003; Díaz-Bautista, 2003; Díaz-Bautista y Celaya Tentori, 2002; Chiquiar, 2005; Esquivel, 2000; Díaz Pedroza, Sánchez Vargas y Mendoza González, 2009; Gómez y Ventosa-Santaularia, 2009), quienes han encontrado la existencia de una desigualdad entre las regiones norte y sur del país; además, los estudios concuerdan generalmente en que existió un largo periodo de convergencia que se modificó en los años ochenta disminuyendo o incluso encontrando divergencia.

Por otro lado, si se asigna al Distrito Federal como la economía líder, existe una convergencia condicionada; es decir, las economías regionales se acercan a ésta, sin embargo, en lugar de que las economías pobres se acerquen más rápidamente a la líder, como predice la teoría de la convergencia, son las economías más ricas las que han logrado aumentar su velocidad y reducir las diferencias entre ellas y la líder, rezagando a las economías pobres y aumentando la desigualdad existente (Díaz Pedroza, Sánchez Vargas y Mendoza González, 2009; Gómez y Ventosa-Santaularia, 2009).

Así pues, el comportamiento de la convergencia puede resumirse en tres periodos: de 1940 a 1970 con una alta velocidad; de 1970 a 1980 con convergencia a tasas muy pequeñas; y finalmente de 1980 en delante con divergencias, aunque esta última etapa se relaciona con el proceso de liberalización económica y apertura comercial (Germán-Soto, 2005). 
A partir del proceso de descentralización de la producción y la apertura comercial se encontró que existe una relación entre los estados con mayores niveles de capital humano, una menor concentración de las actividades agrícolas y una mayor disponibilidad de infraestructura básica con una mayor concentración de plantas industriales grandes (Chiquiar, 2005), lo cual genera espirales acumulativas que dificultan la tarea de reducir estas desigualdades. Es así como el crecimiento y la expansión de la producción que se dio gracias a la apertura comercial no favorecieron la disminución de la pobreza sino que generaron una división entre los estados (Buitrago, 2009).

Además, a pesar del crecimiento que muestran algunos estados -principalmente los manufactureros-, la desigualdad no ha disminuido aun cuando México se convirtió en un país altamente exportador de manufacturas. Durante los periodos de 1970-1985 y 1985-2001, la frontera norte tuvo tasas de crecimiento per cápita de $1.64 \%$ anual para ambos periodos; la región centro-norte, $2.41 \%$ y $1.27 \%$ anual respectivamente; la región centro-sur, $2.17 \%$ y $0.66 \%$; la región sur, $3.55 \%$ y $0.55 \%$; y finalmente, la ciudad de México tuvo tasas de $1.02 \%$ y $1.33 \%$ para cada periodo (Chiquiar, 2005).

Las manufacturas no se han convertido en una fuente de innovación y progreso tecnológico (Ros, 2008), por lo que México padece de una industrialización sin arrastre, sin las infraestructuras necesarias, atraso tecnológico y productividad basada en el uso extensivo del trabajo y la explotación de los recursos naturales (Dautrey, 2008).

De este modo, para disminuir la desigualdad es necesario que el gobierno local-regional participe con políticas públicas adecuadas a las condiciones propias de la problemática de su región (Calderón Villarreal, 2006). Asimismo, el Estado debe participar en la economía con políticas compensatorias que aminoren estas desigualdades (De Hoyos y Lustig, 2009), políticas que él mismo había omitido realizar (Ocegueda Hernández, 2007).

En tales políticas destaca la creación de infraestructura ya que es un elemento en el cual los diferentes niveles de gobierno pueden intervenir a través del gasto público. Así, la dotación de infraestructuras es un elemento que puede ayudar al crecimiento regional, estatal o incluso municipal. Por esta razón, el papel del gobierno como proveedor de infraestructura se vuelve trascendental. Se puede afirmar, entonces, que la dotación de infraestructuras se convierte en un componente determinante de la política regional (Fuentes y Fuentes, 2003). 


\section{La infraestructura en la economía}

La infraestructura es la suma de los materiales institucionales, personales y físicos que sustentan una economía y contribuyen a la remuneración de los factores teniendo en cuenta una asignación oportuna de recursos, es decir, un grado relativamente alto de integración y el nivel más alto posible de los derechos económicos de las actividades (Buhr, 2009).

Buhr (2003 y 2009) reconoce tres tipos de infraestructura, cada una con su propia definición:

1. La infraestructura material se conoce como los bienes de capital que, en forma de equipamiento, facilitan las comunicaciones, transportación, educación, salud y producción mediante el transporte de energía. Es, en esencia, inmóvil y contribuye a la producción de bienes y servicios necesarios para satisfacer los requisitos básicos -físicos y sociales- de los agentes económicos. La función económica de la infraestructura es colaborar para que la producción en masa sea económicamente factible, debido a que su naturaleza física y su costo es inaccesible para los agentes económicos individuales (hogares, empresas, etcétera).

2. La infraestructura institucional incluye todos los hábitos sociales y el establecimiento de las reglas formales y las restricciones informales (convenciones, normas de comportamiento) para dar forma a la mejor interacción humana.

3. La infraestructura personal comprende el número, la estructura y las propiedades relevantes de la población activa -la oferta laboral de una economía-, independientemente de si la fuerza laboral está empleada o desempleada.

Dentro de la infraestructura material también existen otras clasificaciones. Por ejemplo, para Hansen (1965) esta infraestructura puede clasificarse en dos subcategorías: la infraestructura social (SOC) y la infraestructura económica (EOC). Las inversiones públicas específicas clasificadas como EOC son: carreteras; suministro de gas y electricidad; abastecimiento de agua, drenaje y alcantarillado; puentes, puertos y sistemas de transporte fluvial; casas; sistemas de riego; y mercados. Por otra parte, se clasifican como soc escuelas, cuerpos de bomberos, policía, edificios públicos (distintos de los 
incluidos en la EOC), recolección de basura y residuos, servicio postal, parques y campos deportivos, remozamiento de la ciudad, salud, hogares para adultos mayores y vehículos (siempre que no se utilicen para una actividad EOC).

Sin embargo, debido a la amplitud del concepto de infraestructura y de lo diversas que éstas pueden ser, existen problemas para su medición. La cuantificación de las infraestructuras es un problema en el que los expertos no se han puesto de acuerdo, lo que ha dado pie a diferentes métodos. Dependiendo de la información con la que se cuente se pueden dividir, primeramente, en absolutas y relativas. Las primeras se miden en dotaciones absolutas de capital público, mientras que las segundas están relativizadas con algún indicador de tamaño como población o superficie (Fuentes y Mendoza, 2003a).

Físicamente, la infraestructura se mide de dos formas, a saber: en indicadores parciales, es decir, en unidades dependiendo de la categoría en la que se encuentren; ${ }^{1} \mathrm{y}$ en indicadores sintéticos, que se obtienen por categorías de infraestructuras con diferente nivel de agregación por medio de diversos indíces. Monetariamente también se miden de dos maneras: a través de indicadores de flujo, como la formación bruta de capital público por infraestructura; y por indicadores de acervo, los cuales se estiman del acervo de capital que se acumula en las distintas categorías (Fuentes y Mendoza, 2003a).

De este modo, el cálculo de un indicador confiable de infraestructuras permite analizar la distribución de las dotaciones de infraestructura en unidades espaciales, así como la influencia que ésta tiene sobre el crecimiento de los niveles de ingreso (Becerril Torres et al., 2009).

Una vez superada la cuestión de la cuantificación y la construcción de series, el debate se centra en cómo afecta la infraestructura a la actividad económica y cuál es su relación con el crecimiento de la misma.

El efecto de la infraestructura en la actividad económica se da directamente en la función de producción e indirectamente a través de la utilidad de las familias. Lo primero se debe a que la dotación de bienes de capital físico afectan positivamente la productividad (carreteras, aeropuertos, par-

\footnotetext{
1 Así, las carreteras se miden en kilómetros; la electricidad por unidades de medidores, transformadores, estaciones de distribución, etcétera.
} 
ques industriales, etcétera), tanto como la protección judicial de la propiedad intelectual de las empresas, el gasto en educación e investigación en innovación y desarrollo, entre otros. La segunda afecta la función de utilidad de los hogares mediante la dotación de capital físico no productivo (parques, museos, estatuas y monumentos, etcétera) y en gastos que generan utilidad a los individuos, aunque ésta no sea física ni productiva (bodas reales, fiestas públicas, celebraciones patrias, etcétera) (Sala-i-Martin, 2000). Además, el efecto de la infraestructura también impacta en las habilidades y capacidades de los individuos y esto incide indirectamente en la producción (De Haan et al., 2007).

Para Nijkamp y Ubbels (1999), otros efectos de la inversión en infraestructura son:

- Construcción directa y los efectos asociados con la construcción financiada por el gasto público. Estos efectos son generalmente a corto plazo y se acumulan sobre todo en el sector de la construcción, ya sea dentro o fuera de la región.

- Efectos indirectos sobre la eficiencia económica (efectos redistributivos) asociados a las ventajas de los precios relativos de las empresas situadas en una región donde se realiza el capital social fijo (por ejemplo, como resultado de una mejor accesibilidad geográfica o un mejor acceso a los servicios educativos o de investigación). Estos efectos de precios conducen a una mejora de la posición competitiva de las empresas regionales a través de las ganancias del comercio. El argumento básico es que la mejora de la accesibilidad conduce a una reducción en los costos de transporte para las empresas y los hogares.

- Efectos generativos asociados con el nacimiento o la reubicación de nuevas empresas atraídas a la región debido a su posición competitiva mejorada.

\section{La infraestructura en los municipios de la frontera norte de México: evidencia y estrategia empírica}

Felstein y Ha (1995) realizaron el primer trabajo sobre el tema para México. Estos autores basan su análisis en tres tipos de infraestructura: electricidad, transporte y comunicaciones, y encuentran que ellas tienden a reducir el 
costo de la producción sectorial. Sin embargo, respecto de la infraestructura carretera sus hallazgos indican que ésta es en gran parte improductiva y advierten que los resultados pueden no ser confiables debido a la disponibilidad de datos.

Lächler y Aschauer (1998) encuentran evidencia que sugiere que los aumentos en la inversión pública no se traducen automáticamente en un rápido crecimiento de la producción y la productividad. Depende del tipo de inversión tanto como de la intención de ésta.

Fuentes y Mendoza (2003b) señalan que la relación capital público-PIB en el periodo 1980-1985 tiene un efecto positivo y significativo sobre la tasa de crecimiento de México, lo cual es congruente con las políticas redistributivas del gobierno. Sin embargo, a partir del periodo de 1985-1998 se perdieron tanto la intensidad como la significancia de las variables de infraestructura. Esto pudo deberse al cambio de prioridades del gobierno, ahora centrado en la estabilidad macroeconómica. También mencionan que la apertura comercial tuvo un papel determinante, no en cuanto a la asignación presupuestaria en obras de infraestructura, sino en cuanto al apoyo estatal al capital privado para invertir en la construcción y la administración de obras y servicios públicos, lo que hace necesario realizar estudios regionales que midan el impacto en las regiones ganadoras.

En un análisis por regiones de México se encuentra que la infraestructura fue estadísticamente significativa en la explicación de las variaciones regionales del Рів. Se recomienda una política pública sostenida de dotación de infraestructura física productiva para disminuir las desigualdades regionales del país, poniendo énfasis en las necesidades de cada región y no en una política central (Fuentes, 2003).

Finalmente, Noriega y Fontela (2007) descubrieron que los choques en la infraestructura tienen efectos positivos y significativos de largo plazo en ambas medidas de la electricidad y las carreteras. Los resultados indican que, en ambos casos, no se han alcanzado en México los niveles de infraestructura que maximizan el crecimiento económico en el periodo estudiado.

En la frontera norte se observa una realidad compleja y heterogénea, desvinculada muchas veces en los aspectos económicos, sociales, políticos y culturales del centro del país. Es por eso que las políticas centrales no tienen los resultados esperados en dicha zona y surge la necesidad de abordarla con políticas regionales más acordes a su situación. Cabe señalar, además, 
que la región tiene un avanzado grado de crecimiento en comparación con otras regiones del país (Díaz-Bautista, 2003).

La frontera norte abarca seis estados de la república mexicana y, según cifras de INEGI, en 2004 representó 23\% de la producción nacional. En conjunto, la región abarca 274 municipios, de los cuales 35 están sobre la franja fronteriza y representan $25 \%$ de la producción total de los estados de la frontera norte y $6 \%$ de la nacional. De entre estos municipios destacan 11 que concentran $24.5 \%$ de la producción de los estados fronterizos (por ende, la aportación de los 24 municipios restantes es poco significativa).

En vista de que 11 de los 35 municipios explican $95 \%$ de la producción total de los municipios fronterizos, se considera que el estudio de las ciudades capitales corresponde con el de esa demarcación administrativa, quedando así el recorte geográfico de la siguiente forma: por Baja California, Mexicali y Tijuana; por Sonora, Nogales; por Chihuahua, Ciudad Juárez; por Coahuila, Piedras Negras; y por Tamaulipas, Reynosa, Nuevo Laredo y Matamoros. Cabe mencionar que Nuevo León es excluido de la investigación ya que sólo cuenta con un municipio fronterizo y éste representa sólo $0.06 \%$ de la producción del estado.

Sin embargo, la producción también está localizada en unas cuantas ciudades por estado, principalmente en las capitales, de forma que también es importante analizar el comportamiento de las ciudades principales. Es por ello que en el análisis se incluyen: Hermosillo y Ciudad Obregón por Sonora; Chihuahua por la entidad del mismo nombre; Saltillo, Ramos Arizpe y Torreón por Coahuila; y finalmente Tampico por Tamaulipas. Dichas ciudades -también llamadas "de segunda frontera"- se vieron beneficiadas por la apertura comercial y tienen una dinámica menor que la de las fronteras, pero con una seria relación entre éstas (Castro, 2007).

Debido a lo complejo de la obtención de datos, los diferentes métodos presentan ventajas y desventajas. Para el caso de México, y en particular por lo que toca a los municipios, la información no es regular ni consistente, por lo consideramos que el método de índices es el más apropiado para esta investigación.

El primer paso consistió en agrupar la información por categorías, para lo cual, siguiendo a Hansen, Aschauer y Bhiel, dividimos las infraestructuras en dos tipos: aquellas que ayudan al crecimiento de la productividad y aquellas que contribuyen al crecimiento económico. 
Se obtuvo así un Índice Global de Infraestructura Productiva (IGP), compuesto por dos categorías: la Infraestructura Productiva Social (IPS), representada por el número de escuelas; y la Infraestructura Productiva Económica (IPE) que agrupa tres subcategorías:2

- Infraestructura energética (IE), compuesta por las siguientes variables: fuentes de abastecimiento de agua (pozos), tomas eléctricas domiciliarias (unidades), transformadores de distribución (unidades), longitud de líneas $(\mathrm{km})$.

- Infraestructura de transportes (IT), compuesta por: red de carreteras principales $(\mathrm{km})$, secundarias $(\mathrm{km})$, rurales $(\mathrm{km})$, camiones de carga (unidades), aeropuertos (unidades) y vuelos comerciales (unidades). ${ }^{3}$ Infraestructura de comunicaciones (IC), compuesta por: oficinas de telégrafos (unidades), oficinas postales (unidades) y televisión por cable (conexiones). ${ }^{4}$

- La frontera norte ha experimentado tasas de crecimiento poblacional por encima del promedio nacional, por lo que el crecimiento de la población es un elemento importante para el crecimiento de las infraestructuras. Para omitir este efecto, se obtuvieron las variables per cápita dividiendo cada variable entre la población tomando el periodo de 1993 a 2005 (Alvarez Ayuso y Becerril, 2008; Fuentes, 2003; Fuentes y Mendoza, 2003).

\footnotetext{
2 Se decidió agrupar tres categorías tomando en cuenta que los equipamientos de transportes representan un factor de accesibilidad y permiten organizar la localización de las actividades económicas; las comunicaciones son esenciales para la expansión y la competitividad de las actividades (Becerril Torres et al., 2009); y la provisión de energía eléctrica es un insumo en cualquier tipo de actividad.

3 Se incorporan los vuelos comerciales y el número de camiones de carga como una variable de aproximación a la capacidad de las carreteras y aeropuertos.

4 No se cuenta con información municipal para los años de estudio de líneas telefónicas y conexiones a internet, por esta razón, en su lugar se utiliza la televisión por cable como una aproximación a dichas variables.
} 


\section{Cuadro 1. Variables}

\begin{tabular}{|c|c|c|c|}
\hline Índice general & Categoría & Subcategoría & Variables \\
\hline \multirow{4}{*}{$\begin{array}{l}\text { Índice Global } \\
\text { de Infraestructura } \\
\text { Productiva (IGP) }\end{array}$} & $\begin{array}{l}\text { Infraestructura } \\
\text { Productiva } \\
\text { Social (IPS) }\end{array}$ & & $\begin{array}{l}\text { Escuela elemental (EE) } \\
\text { Escuela primaria (EP) } \\
\text { Capacitación para el trabajo (CPT) } \\
\text { Escuela secundaria (ES) } \\
\text { Escuela profesional media (EPM) } \\
\text { Escuela medio superior (EMS) }\end{array}$ \\
\hline & \multirow{3}{*}{$\begin{array}{l}\text { Infraestructura } \\
\text { Productiva } \\
\text { Económica (IPE) }\end{array}$} & $\begin{array}{l}\text { Infraestructura } \\
\text { Energética (IE) }\end{array}$ & $\begin{array}{l}\text { Fuentes de abastecimiento } \\
\text { de agua (FAA) } \\
\text { Tomas eléctricas domiciliarias } \\
\text { (TTE) } \\
\text { Transformadores de distribución } \\
\text { (TRA) } \\
\text { Longitud de líneas (LLE) }\end{array}$ \\
\hline & & $\begin{array}{l}\text { Infraestructura } \\
\text { de Transportes (IT) }\end{array}$ & $\begin{array}{l}\text { Carreteras principales (RCP) } \\
\text { Carreteras secundarias (RCS) } \\
\text { Carreteras rurales (RCR) } \\
\text { Camiones de carga (CC) } \\
\text { Aeropuertos (AE) } \\
\text { Vuelos comerciales (Vc) }\end{array}$ \\
\hline & & $\begin{array}{l}\text { Infraestructura de } \\
\text { Comunicaciones } \\
\text { (Ic) }\end{array}$ & $\begin{array}{l}\text { Oficinas de telégrafos (TG) } \\
\text { Oficinas postales (OPS) } \\
\text { Televisión por cable (TVC) }\end{array}$ \\
\hline
\end{tabular}

Fuente: Elaboración propia.

Adicionalmente, a fin de homogeneizar los distintos tipos de datos y sus respectivas mediciones, se utilizó una normalización convirtiendo las variables en magnitudes adimensionales en forma de porcentaje con respecto al máximo valor de cada variable, teniendo así índices que van de 0 a 100, donde 100 será el municipio con mayor dotación de infraestructura según la variable que se esté tomando como referencia. Formalmente, el cálculo sería:

$$
S_{j, r}=\left(a_{j, r} / a_{\mathrm{MAX}, r}\right) \cdot 100
$$

Donde: 
$a_{j, r}=$ equipamiento de infraestructura para cada variable $j$ en la región $r$.

$a_{\mathrm{MAx}, r}=$ medida de la región con el valor máximo.

$S_{j, r}=$ indicador normalizado para la región $r$ y variable $j$.

Teniendo así valores unidimensionales y comparables dentro de la región. Finalmente, para la agregación de los índices se utilizó el método de Indicadores Sintéticos de Biehl (мB) (Cancelo de la Torre y Uriz Tomé, 1994). Este índice parte del supuesto de que las menores dotaciones en una categoría pueden ser compensadas con mayores dotaciones en alguna otra de las categorías. La agregación se hace con medias aritméticas cuando los equipamientos se consideran como sustitutivos y con medias geométricas cuando no lo son (Fuentes y Mendoza, 2003a).

Se construye entonces cada categoría con una media aritmética de la siguiente forma:

$$
I_{i, r}=(1 / n) \cdot \sum S_{j, r}
$$

Donde:

$I_{i, r}=$ indicador de la categoría en la región.

$S_{j, r}=$ indicador de la subcategoría que está incluido en la categoría.

Las categorías son agregadas con una media geométrica ya que son insustituibles. La formulación es la siguiente:

$$
I G_{r}=\sqrt[\mathrm{n}]{\prod_{i}^{\mathrm{n}} I_{i, r}}
$$

Donde:

$I G_{r}=$ Indicador global de infraestructura en la región $r$.

$I_{i, r}=$ Indicador de la categoría en la región $r$. 


\section{Cuadro 2. Índice Global de Infraestructuras con мв en valores per cápita 1993-2005}

\begin{tabular}{|c|c|c|c|c|c|c|c|c|c|c|c|c|c|}
\hline & ஜ & ஓ & $\stackrel{\text { 오 }}{\circ}$ & ஃ & $\stackrel{\hat{\circ}}{\circ}$ & $\stackrel{\circ}{\stackrel{\circ}{\circ}}$ & $\stackrel{\text { ஜ }}{\stackrel{一}{\circ}}$ & ঃั & চ্ণ & ণั & ֻั & ষ্ণ & นొ \\
\hline Mexicali & 56 & 55 & 57 & 61 & 62 & 63 & 63 & 65 & 57 & 52 & 53 & 55 & 58 \\
\hline Tijuana & 23 & 24 & 30 & 30 & 28 & 30 & 28 & 26 & 27 & 26 & 27 & 28 & 29 \\
\hline Piedras Negras & 60 & 58 & 60 & 63 & 63 & 65 & 64 & 67 & 63 & 64 & 63 & 64 & 62 \\
\hline Saltillo & 43 & 40 & 43 & 46 & 45 & 47 & 49 & 52 & 47 & 47 & 46 & 45 & 44 \\
\hline Torreón & 38 & 37 & 40 & 41 & 48 & 50 & 49 & 43 & 47 & 47 & 46 & 48 & 48 \\
\hline Chihuahua & 41 & 41 & 41 & 42 & 46 & 48 & 52 & 55 & 49 & 46 & 45 & 47 & 47 \\
\hline Juárez & 27 & 25 & 23 & 23 & 25 & 27 & 28 & 29 & 25 & 24 & 22 & 25 & 25 \\
\hline Cajeme & 51 & 56 & 53 & 54 & 55 & 59 & 59 & 63 & 58 & 57 & 57 & 61 & 64 \\
\hline Hermosillo & 47 & 56 & 52 & 55 & 58 & 60 & 62 & 67 & 59 & 58 & 55 & 55 & 53 \\
\hline Nogales & 56 & 64 & 58 & 60 & 59 & 58 & 57 & 63 & 57 & 57 & 54 & 51 & 54 \\
\hline Matamoros & 35 & 34 & 36 & 37 & 39 & 42 & 42 & 44 & 45 & 46 & 44 & 46 & 46 \\
\hline Nuevo Laredo & 30 & 29 & 33 & 34 & 33 & 32 & 38 & 41 & 39 & 39 & 38 & 39 & 39 \\
\hline Reynosa & 32 & 28 & 34 & 35 & 32 & 38 & 40 & 39 & 42 & 40 & 38 & 39 & 40 \\
\hline Tampico & 36 & 36 & 38 & 38 & 38 & 41 & 42 & 48 & 45 & 42 & 45 & 47 & 46 \\
\hline
\end{tabular}

Fuente: Elaboración propia.

Se aprecia una disparidad en el caso de Baja California entre Tijuana y Mexicali, mientras que Coahuila, Saltillo y Torreón tienen dotaciones similares y Piedras Negras tiene la mayor infraestructura. Para el caso del estado de Chihuahua hay una disparidad que favorece a la capital. Para Sonora y Tamaulipas, las dotaciones son similares entre sus municipios. 


\section{Cuadro 3. Índice de Infraestructura Productiva Económica con мв en valores per cápita 1993-2005}

\begin{tabular}{|c|c|c|c|c|c|c|c|c|c|c|c|c|c|}
\hline & ஜㅇ & ஓ & 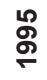 & $\stackrel{\circ}{\circ}$ & 홍 & $\stackrel{\circ}{\circ}$ & $\stackrel{\circ}{\circ}$ & ঃั & চ & ণั & ஜั & ర్ & 옹 \\
\hline Mexicali & 53 & 50 & 54 & 60 & 60 & 62 & 63 & 64 & 54 & 48 & 51 & 53 & 56 \\
\hline Tijuana & 18 & 18 & 25 & 25 & 24 & 25 & 24 & 21 & 22 & 21 & 23 & 23 & 25 \\
\hline Piedras Negras & 57 & 53 & 57 & 60 & 61 & 65 & 63 & 66 & 61 & 63 & 61 & 63 & 61 \\
\hline Saltillo & 35 & 32 & 35 & 39 & 39 & 40 & 42 & 45 & 39 & 40 & 40 & 38 & 37 \\
\hline Torreón & 30 & 28 & 32 & 33 & 40 & 42 & 41 & 34 & 38 & 38 & 38 & 40 & 39 \\
\hline Chihuahua & 35 & 35 & 35 & 36 & 40 & 42 & 46 & 49 & 43 & 40 & 40 & 43 & 43 \\
\hline Juárez & 24 & 22 & 19 & 20 & 22 & 23 & 24 & 25 & 21 & 20 & 18 & 20 & 21 \\
\hline Cajeme & 45 & 49 & 47 & 48 & 49 & 52 & 53 & 60 & 52 & 52 & 53 & 57 & 61 \\
\hline Hermosillo & 43 & 50 & 46 & 50 & 53 & 55 & 58 & 64 & 56 & 55 & 52 & 51 & 49 \\
\hline Nogales & 55 & 60 & 53 & 57 & 56 & 54 & 54 & 61 & 53 & 53 & 49 & 49 & 52 \\
\hline Matamoros & 32 & 30 & 33 & 33 & 36 & 39 & 39 & 42 & 42 & 44 & 43 & 44 & 44 \\
\hline Nuevo Laredo & 25 & 24 & 29 & 30 & 28 & 28 & 35 & 38 & 37 & 35 & 35 & 36 & 36 \\
\hline Reynosa & 27 & 22 & 29 & 31 & 28 & 35 & 37 & 36 & 40 & 37 & 36 & 36 & 37 \\
\hline Tampico & 29 & 29 & 31 & 31 & 31 & 34 & 35 & 42 & 39 & 36 & 40 & 42 & 40 \\
\hline
\end{tabular}

Fuente: Elaboración propia.

El cuadro 3 muestra cómo en relación con la infraestructura productiva, Baja California y Chihuahua aumentan aún más las desigualdades en sus dotaciones respecto de los demás estados, ya que la dotación de éstos permanece similar en el tiempo mientras que en los demás existe un crecimiento en el índice global. 
Para conocer la relación de la infraestructura con el crecimiento económico es necesario construir un modelo económico que relacione el ingreso per cápita como variable dependiente con el capital, el trabajo y las variables de infraestructura elegidas. Para ello se utilizó el método de panel de datos, modelo que dispone simultáneamente de información de corte transversal y de serie temporal (Greene, 2002).

Existen características para cada individuo que influyen sobre la variable dependiente y que se mantienen fijas en el tiempo; si no se toman en cuenta, se incurre en un sesgo. El método de panel de datos considera las heterogeneidades inherentes a cada individuo con la variable dependiente (Wooldridge, 2002).

Un panel de datos proporciona menos problemas de multicolinealidad, más grados de libertad y, por lo tanto, mayor eficiencia. A su vez, permite utilizar un conjunto de datos más informativos, en el sentido de que es capaz de recoger con mayor precisión la variabilidad en los datos, tanto la existente entre individuos como a lo largo del tiempo (De la Fuente, 2008). Finalmente, un panel de datos recoge a nivel de microunidades como individuos, empresas u hogares y, en nuestro caso, municipios, eliminando los sesgos causados por la agregación.

Un panel de datos puede ser formulado de diferentes maneras:

- Los coeficientes de las pendientes son constantes, pero el término independiente varía entre individuos.

- Los coeficientes de las pendientes son constantes, pero el término independiente varía entre individuos y tiempo.

- Todos los coeficientes varían entre individuos.

- Todos los coeficientes varían entre individuos y tiempo.

En todos los anteriores se asume que el término de error tiene una distribución idéntica e independiente sobre individuos y sobre el tiempo, con media cero y varianza mínima (Cobacho Tornel y Bosch Mossi, 2005).

El modelo de efectos fijos considera que existe un término constante diferente para cada individuo y supone que los efectos individuales son independientes entre sí. Con este modelo se considera que las variables explicativas afectan por igual a las unidades de corte transversal y que éstas se diferencian por características propias de cada una de ellas, medidas por medio del intercepto o de la variable elegida (Mayorga y Muñoz, 2000). 
En contraste, el modelo de efectos aleatorios considera que los efectos individuales no son independientes entre sí, sino que están distribuidos aleatoriamente alrededor de un valor dado.

Para esta investigación supondremos tres clases de modelos: a) los coeficientes son iguales para todos los municipios, es decir, el comportamiento es el mismo para todos los municipios y suponemos una misma función de producción; b) los coeficientes del trabajo, el capital y las variables de infraestructura son iguales para todos los municipios y las pendientes son diferentes para cada municipio, lo cual nos indica una misma función de producción para éstos, sin embargo, se toman en cuenta efectos propios de la región que pueden modificar la producción; y c) los coeficientes del trabajo y el capital son iguales para todos los municipios y las pendientes y las variables de infraestructura son diferentes para cada municipio, es decir, se tiene la misma función de producción pero el efecto de la infraestructura es diferente en cada región debido a particularidades propias de cada municipio.

El modelo supone una relación del tipo $y=f(k, L, V I)$ donde $y$ es el ingreso per cápita, VI son las variables de la infraestructura, $k$ el capital por trabajador y $L$ el trabajo industrial y de servicios. Para ello se necesitaron las series de datos de un periodo de 1993 a 2005.

Las series de ingreso municipal, población y activos fijos no cuentan con información anual, por lo tanto, fue necesario estimarlas mediante los siguientes métodos:

\section{Ingreso municipal}

Para la estimación del nivel de ingreso por municipio se utiliza la metodología planteada por Gerber (2003) donde $Y m$ se estima a partir del ingreso estatal Ys, ingreso estatal Ysi en el sector $i$, el empleo municipal $e_{m i}$ en el sector $i$ y el empleo estatal esi en el sector $i$. Entonces:

$$
\begin{aligned}
& Y_{m}=\lambda Y_{s} \quad \text { donde } \quad 0<\lambda<1, \\
& \lambda=\sum_{i}\left(\left(Y_{s i} / Y_{s}\right)\left(e_{m i} / e_{s i}\right)\right)
\end{aligned}
$$

Los activos fijos se estiman de la misma forma. 


\section{Población}

La población se obtiene mediante una interpolación siguiendo la metodología utilizada por Mendoza (2006, 2006a), donde se aplica la siguiente fórmula para obtener los datos intercensales.

$$
P_{m, t+1}=\left(\left(P_{m, t} \bullet e^{a}\right)\left(\frac{\operatorname{In}\left(\frac{P_{m, t+n}}{P_{m, t}}\right)}{n}\right)\right.
$$

Donde:

$P=$ Población

$a=\mathrm{año}$

$t=$ año base

\section{Participación indirecta de la infraestructura en la producción}

En el cuadro 4 se muestra en las tres primeras estimaciones en el orden mencionado la inclusión del IGP como un factor que afecta la producción indirectamente, es decir, en una estimación lineal. En el primer caso, el coeficiente del IGP no es significativo. La producción no se explica por la infraestructura, sin embargo, estos resultados se revierten al incluir la hipótesis de la heterogeneidad municipal e insertar los efectos fijos en la estimación. El resultado es el esperado: con un mayor peso en el factor trabajo sobre el capital por ser municipios de economías basadas en la maquiladora de exportación, el IGP es elevado, significativo y positivo. En la tercer estimación, con efectos fijos para el IGP, los coeficientes de trabajo y capital permanecen constantes; los componentes del IGP en los municipios son mayormente positivos, pero no significativos, excepto para el caso de Piedras Negras, Torreón y Matamoros. 


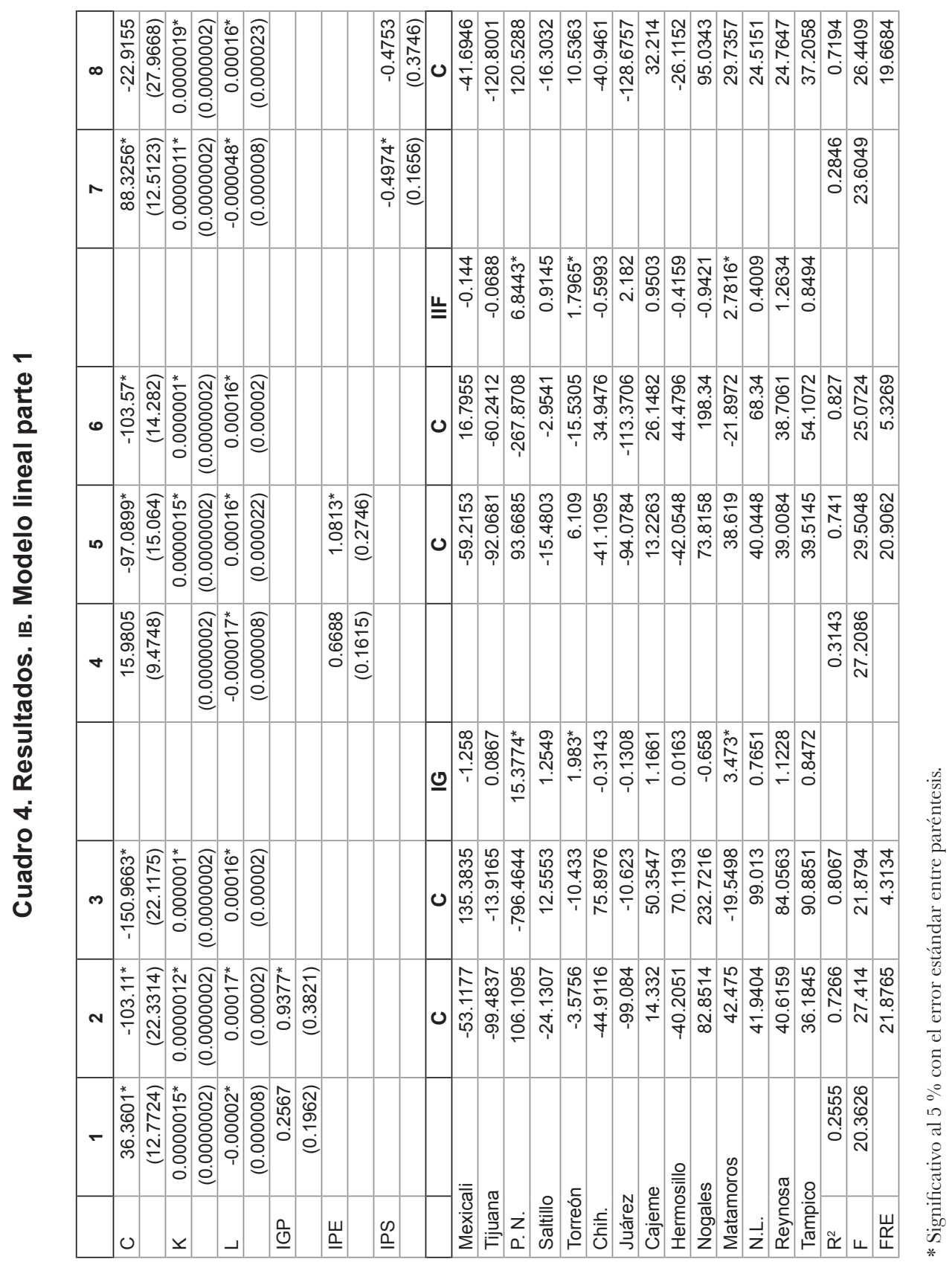


El siguiente modelo es el que incluye la variable que nos ocupa, el IPE, que en la estimación homogénea presenta un coeficiente significativo y positivo. Con efectos fijos, dicho coeficiente mejora significativamente, permanece positivo y estadísticamente válido. Al incluir el índice a la hipótesis de heterogeneidad municipal, el capital y el trabajo permanecen constantes. Los efectos fijos presentan mayormente elementos positivos y de éstos sólo tres son estadísticamente significativos, permaneciendo entre ellos Piedras Negras, Torreón y Matamoros.

En el tercer modelo con la inclusión del IPS, la estimación homogénea del coeficiente es negativa y significativa.

La estimación con efectos fijos en la constante no cumple con la hipótesis de FRE y no se toma en cuenta; con efectos fijos en el IPS, los coeficientes son mayormente negativos y tres son significativos.

En el cuadro 5 se hace una estimación contrastando el IPE con el IPs. Bajo el supuesto de homogeneidad, todas las variables son significativas y positivas con excepción del IPS. Al introducir los efectos fijos, el coeficiente del IPE aumenta, mientras que el del IPS disminuye. Suponiendo heterogeneidad municipal, los coeficientes del IPE son mayormente positivos y significativos para Piedras Negras y Matamoros, mientras que para el IPS son mayormente negativos y significativos para Ciudad Juárez y Reynosa, presentándose una relación inversa en nueve municipios.

Finalmente, se incluye un modelo con los IE, IT e IC en la estimación homogénea. Los tres índices son significativos, positivos en IE e IC y negativo en IT. La variable del trabajo no es significativa. Las subcategorías presentan las mismas relaciones que se habían observado en la estimación de los indicadores parciales, con una relación negativa en transportes y un mayor coeficiente en la energía. Al introducir los efectos fijos el Iт deja de ser significativo. Los dos índices restantes tienen menos poder explicativo. El trabajo se vuelve significativo. El modelo con heterogeneidad municipal no cumple con la hipótesis de no efectos redundantes, por lo que no es tomado en cuenta.

\section{Participación directa de la infraestructura en la producción}

Para obtener estos modelos se aplican logaritmos a todas las variables -a excepción de los índices- y se hacen las mismas estimaciones. 


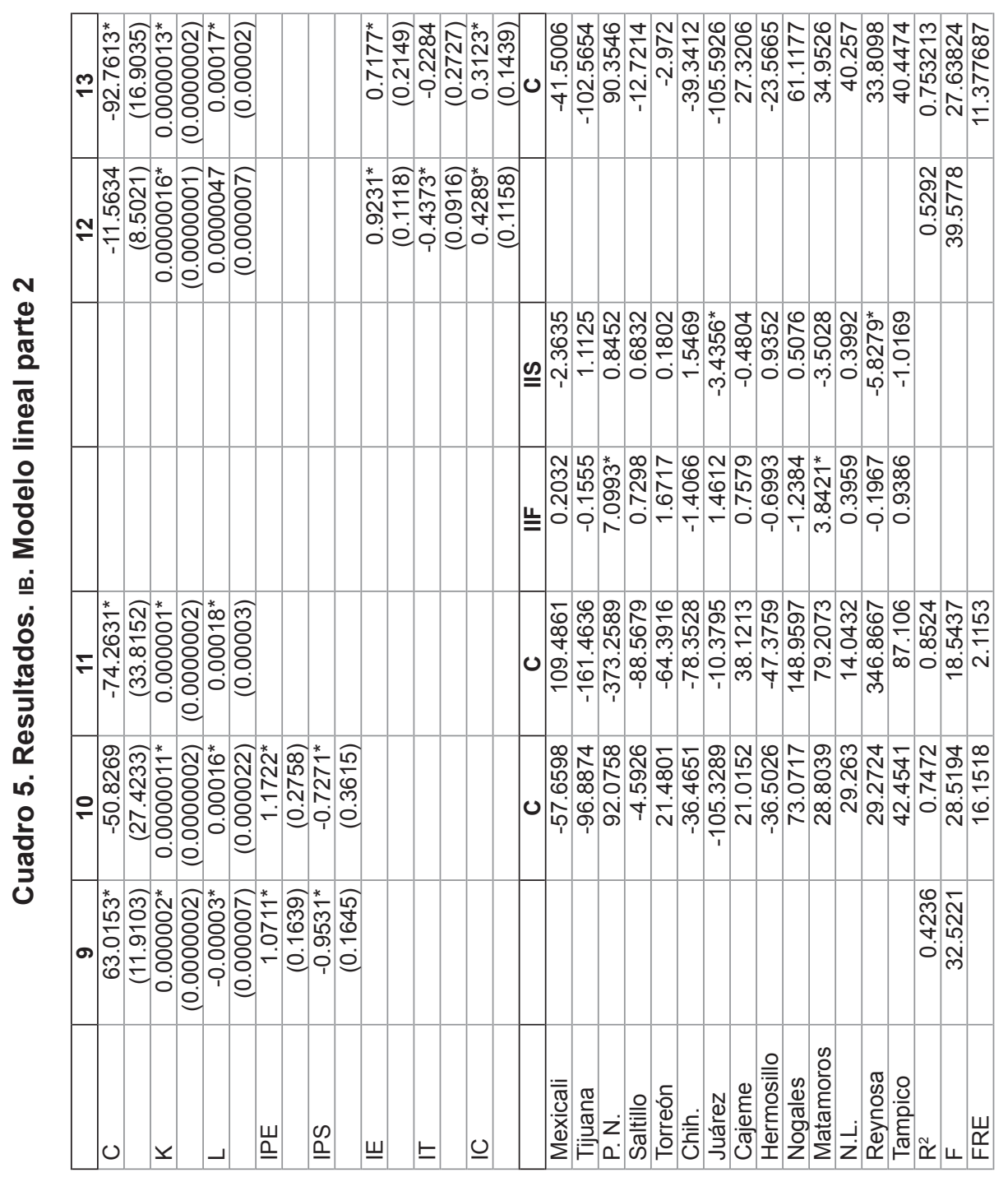

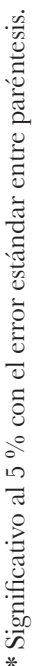


El cuadro 6 presenta en sus primeras tres estimaciones al IGP. En la primera estimación, bajo el supuesto de homogeneidad, las cuatro variables son significativas; sin embargo, el IGP y el trabajo presentan una relación negativa. En la segunda estimación, al introducir los efectos fijos se revierten estos dos coeficientes; el trabajo cobra mayor relevancia al pasar a estimaciones con efectos fijos y heterogeneidad municipal en los índices. Sin embargo, el índice global de infraestructura no es significativo. Con heterogeneidad municipal en los índices, el modelo es significativo en sus variables explicativas y en las variables municipales. Ahora son cuatro significativos en comparación con el modelo lineal: Nogales y Matamoros permanecen, se agregan Torreón (positivo) y Reynosa (negativo).

Con respecto al IPE, en la primera estimación aparece como no significativo. En la estimación con efectos fijos es positivo y significativo; en la tercera estimación, los estimadores del índice son mayormente positivos y es el modelo que mayor número de municipios significativos presenta (seis), entre ellos Nogales y Piedras Negras -los de mayor valor-, y Tijuana y Ciudad Juárez-que presentan un valor positivo y significativo-. Recordemos que Tijuana había presentado relaciones negativas en las estimaciones de indicadores parciales y en las estimaciones lineales.

En contraste, el modelo con el IPS sigue presentando estimadores negativos. En la segunda estimación no es significativo y en la tercera los estimadores municipales significativos son negativos a excepción de Nogales. La relación supone que a medida que aumentan las escuelas disminuyen los trabajadores disponibles para las industrias.

En el tercer modelo se incluyen las dos subcategorías. Para la primera estimación, el trabajo y el IPs tienen una relación negativa. Todas las variables son significativas. Al introducir los efectos fijos en el intercepto, el trabajo se vuelve positivo y es el elemento con mayor peso en la regresión. La variable IPs pierde significancia. El IPE permanece positivo y significativo, lo que confirma su papel determinante en el crecimiento de las economías. En la tercera estimación aparecen cinco municipios estadísticamente significativos con elevados valores positivos. Sólo Reynosa manifiesta una relación inversa. 


\begin{tabular}{|c|c|c|c|c|c|c|c|c|c|c|c|c|c|c|c|c|c|c|c|c|c|c|c|c|c|c|c|c|}
\hline & & & & & & & & & & & & $\begin{array}{l}\varrho \\
\underline{\underline{z}} \\
\mathbf{z}\end{array}$ & 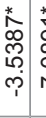 & '. & & & 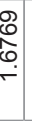 & \begin{tabular}{l}
$m$ \\
\hdashline \\
0 \\
0 \\
0 \\
\end{tabular} & 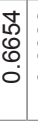 & 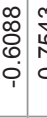 & & 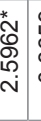 & 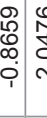 & 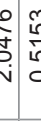 & $\frac{\hat{N}}{\stackrel{N}{N}}$ & & & \\
\hline$\sigma$ & 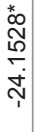 & 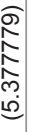 & 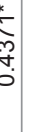 & 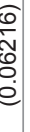 & 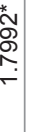 & 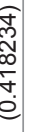 & & & & & & u & 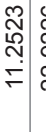 & 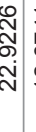 & 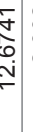 & ָָ & 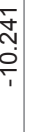 & $\begin{array}{c}0 \\
0 \\
0 \\
0 \\
\varphi \\
1\end{array}$ & $\begin{array}{c}\hat{\infty} \\
\bar{c} \\
\varphi \\
i\end{array}$ & 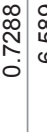 & & 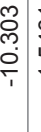 & 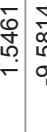 & 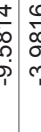 & $\stackrel{0}{\frac{0}{n}}$ & \begin{tabular}{|c|}
$\infty$ \\
0 \\
$\mathscr{0}$ \\
$\infty$ \\
0 \\
\end{tabular} & 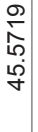 & $\begin{array}{l}\frac{\infty}{\leftarrow} \\
\stackrel{\sigma}{+}\end{array}$ \\
\hline$\infty$ & $\begin{array}{l}\text { * } \\
\stackrel{\text { ph }}{\rho} \\
\text { m. } \\
\stackrel{p}{p} \\
1\end{array}$ & 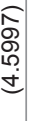 & 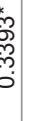 & 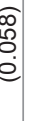 & 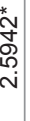 & 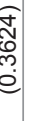 & & & & \begin{tabular}{l}
0 \\
\multirow{2}{0}{} \\
0 \\
0 \\
0
\end{tabular} & 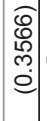 & ט & 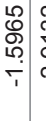 & స్ & 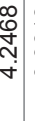 & 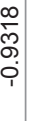 & 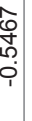 & 察 & 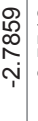 & 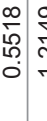 & זִָ & $\begin{array}{l}0 \\
0 \\
0 \\
\vdots \\
\dot{m}\end{array}$ & 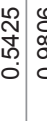 & 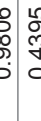 & 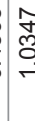 & $\mid \begin{array}{l}0 \\
f \\
\infty \\
0 \\
0\end{array}$ & $\begin{array}{l}\hat{0} \\
\hat{o} \\
0 \\
0 \\
i\end{array}$ & $\frac{\mathbb{N}}{\stackrel{N}{\sigma}}$ \\
\hline$\wedge$ & 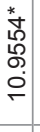 & 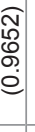 & م. & $\begin{array}{l}0 \\
0 \\
0 \\
\dot{e} \\
\dot{e}\end{array}$ & 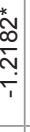 & 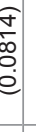 & & & & 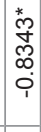 & $\mid \begin{array}{c}\widehat{\Omega} \\
\hat{n} \\
\stackrel{0}{0} \\
\end{array}$ & & & & & & & & & & & & & & & \begin{tabular}{|l|}
$\infty$ \\
\hdashline \\
0 \\
0 \\
0 \\
$\dot{0}$
\end{tabular} & 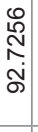 & \\
\hline & & & & & & & & & & & & $\begin{array}{l}\text { ul } \\
\text { z }\end{array}$ & $\begin{array}{l}\mathrm{O} \\
0 \\
0 \\
0\end{array}$ & 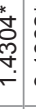 & ț & 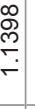 & ז & 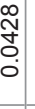 & 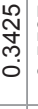 & 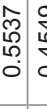 & 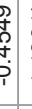 & 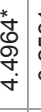 & 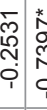 & 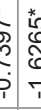 & $\begin{array}{l}\bar{\delta} \\
\text { do } \\
0\end{array}$ & & & \\
\hline 0 & 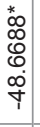 & $\begin{array}{l}\overparen{f} \\
\stackrel{+}{+} \\
\dot{ \pm}\end{array}$ & 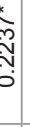 & $\begin{array}{l}\hat{e} \\
\tilde{e} \\
\hat{e} \\
\dot{e}\end{array}$ & 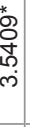 & $\begin{array}{l}\infty \\
\text { o } \\
\\
e \\
e\end{array}$ & & & & & & 0 & 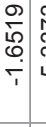 & 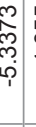 & 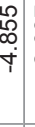 & 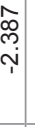 & 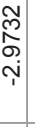 & 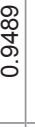 & 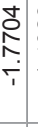 & 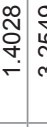 & S. & 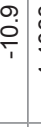 & 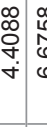 & 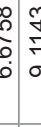 & $\left\{\begin{array}{l}l \\
8 \\
0 \\
\vdots \\
\forall\end{array}\right.$ & \begin{tabular}{|l|} 
\\
0 \\
0 \\
0 \\
0 \\
\end{tabular} & 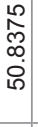 & $\begin{array}{l}0 \\
\infty \\
0 \\
0 \\
\sim \\
\sim\end{array}$ \\
\hline is & $\begin{array}{l}* \\
8 \\
8 \\
8 \\
0 \\
p \\
1\end{array}$ & 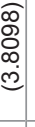 & 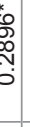 & $\begin{array}{l}8 \\
8 \\
8 \\
\vdots \\
0\end{array}$ & 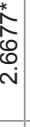 & 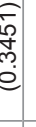 & & 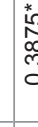 & 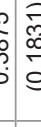 & & & 0 & 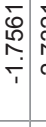 & ָָ & $\begin{array}{l}\infty \\
8 \\
\\
\\
+\end{array}$ & 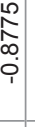 & 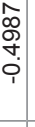 & 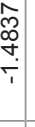 & 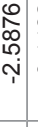 & 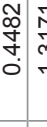 & 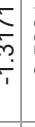 & 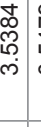 & 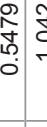 & 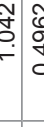 & $\left\{\begin{array}{l}2 \\
0 \\
0 \\
0\end{array}\right.$ & \begin{tabular}{|c|c|}
0 \\
0 \\
$\vdots$ \\
$\infty$ \\
0 \\
$\dot{0}$
\end{tabular} & 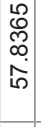 & 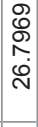 \\
\hline$\nabla$ & 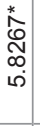 & 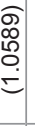 & 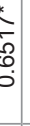 & $\begin{array}{l}\frac{E}{E} \\
0 \\
\dot{0} \\
e\end{array}$ & 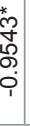 & $\begin{array}{l}\infty \\
8 \\
8 \\
0 \\
0 \\
\end{array}$ & & $\begin{array}{l}\infty \\
\text { cै } \\
\text { है } \\
c\end{array}$ & $\begin{array}{l}: \\
b \\
b \\
:\end{array}$ & & & & & & & & & & & & & & & & & 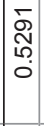 & $\begin{array}{l}10 \\
\delta \\
0 \\
0 \\
0\end{array}$ & \\
\hline & & & & & & & & & & & & $\begin{array}{l}0 \\
z \\
-1\end{array}$ & $\begin{array}{l}0 \\
0 \\
0 \\
0 \\
\vdots \\
1\end{array}$ & 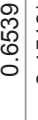 & 老 & 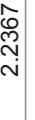 & 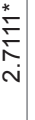 & 告 & \begin{tabular}{c}
$\infty$ \\
$\widetilde{O}$ \\
\hdashline \\
$\vdots$ \\
$\vdots$
\end{tabular} & 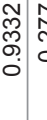 & ș & 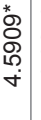 & 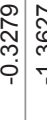 & 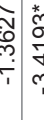 & $\begin{array}{l}8 \\
\tilde{\pi} \\
0 \\
0\end{array}$ & & & \\
\hline$m$ & 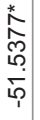 & 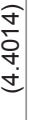 & 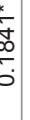 & $\begin{array}{l}\overline{1} \\
0 \\
0 \\
0 \\
0\end{array}$ & 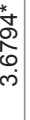 & $\begin{array}{l}\frac{\sigma}{0} \\
\text { ch } \\
e \\
e\end{array}$ & & & & & & 0 & $\begin{array}{l}0 \\
0 \\
\tilde{J} \\
\vdots \\
\dot{\nabla}\end{array}$ & 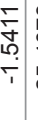 & 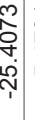 & 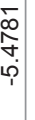 & 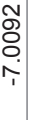 & $\begin{array}{l}\frac{\Sigma}{\delta} \\
\infty \\
0 \\
0\end{array}$ & ָ̊o & 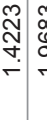 & : & $\begin{array}{l}\hat{N} \\
\tilde{\alpha} \\
\infty \\
\dot{p}\end{array}$ & 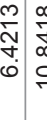 & 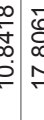 & 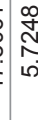 & 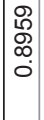 & 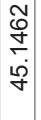 & $\begin{array}{l}\mathcal{N} \\
\tilde{S} \\
\sigma \\
\dot{\sim}\end{array}$ \\
\hline$N$ & $\begin{array}{l}\text { * } \\
0 \\
0 \\
10 \\
\infty \\
\oplus \\
1\end{array}$ & $\begin{array}{c}\stackrel{-}{0} \\
\stackrel{ \pm}{ \pm}\end{array}$ & 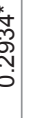 & $\begin{array}{l}\infty \\
\frac{\infty}{8} \\
\dot{0}\end{array}$ & 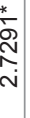 & 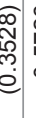 & 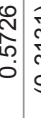 & & & & & 0 & 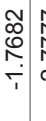 & $\begin{array}{l}\hat{N} \\
\hat{N} \\
\text { i }\end{array}$ & 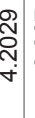 & 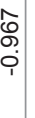 & 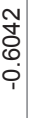 & 足 & 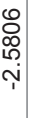 & 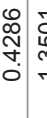 & 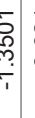 & $\begin{array}{l}\bar{ָ} \\
\dot{\omega}\end{array}$ & 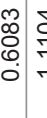 & 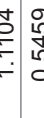 & 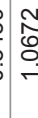 & 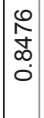 & $\begin{array}{l}\bar{\delta} \\
\dot{o} \\
\\
\omega\end{array}$ & 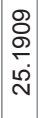 \\
\hline- & 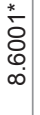 & 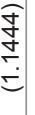 & 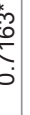 & $\frac{15}{5}$ & . & 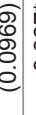 & 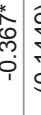 & & & & & & & & & & & & & & & & & & & $\mid \begin{array}{c}N \\
\tilde{f} \\
\tilde{w} \\
0 \\
0\end{array}$ & 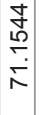 & \\
\hline & 0 & & $\begin{array}{r}x \\
z \\
z\end{array}$ & & $\vec{z}^{\prime}$ & & $\begin{array}{l}\text { O) } \\
\text { z }\end{array}$ & - & z & $\begin{array}{l}0 \\
z \\
z\end{array}$ & & & $\begin{array}{l}\overline{\bar{\pi}} \\
\overline{\frac{d}{x}} \\
\bar{\Sigma} \\
\sum\end{array}$ & 离 & $\begin{array}{l}z \\
0\end{array}$ & 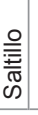 & | & 它 & 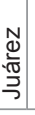 & 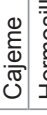 & 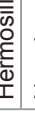 & 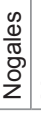 & 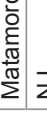 & 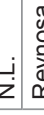 & $=\frac{8}{\frac{0}{2}}$ & $\tilde{\check{x}}$ & ૫ & 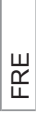 \\
\hline
\end{tabular}




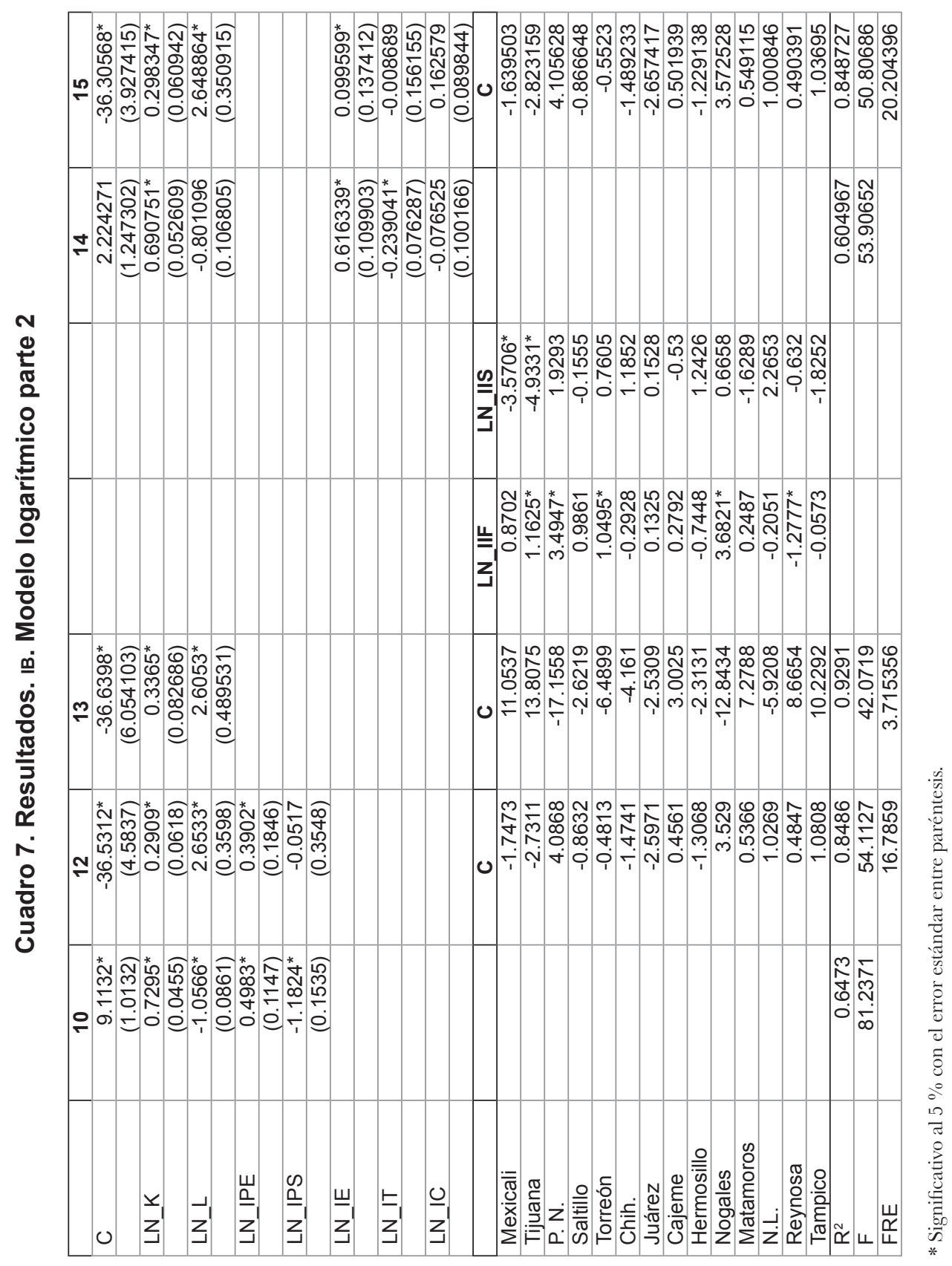


Finalmente, en la primera estimación del último modelo, sólo el capital y las subcategorías de energía y de transporte son significativos. Al introducir los efectos fijos en la segunda estimación, las tres subcategorías dejan de ser significativas; es decir, ninguna de las tres explica a la variable dependiente. En la tercera estimación no se cumple con la hipótesis de efectos no redundantes.

Los indicadores de infraestructura no dicen nada por sí mismos ya que están afectados por el tamaño del municipio y el tamaño de la población. Cuando se transforman las variables a valores unidimensionales y se agregan en dos diferentes índices se obtienen un mayor poder explicativo. Sin embargo, el efecto de la población sigue estando presente, por lo que al poner los índices en términos per cápita tenemos indicadores más confiables y con valores similares en ambos métodos.

Los modelos logarítmicos tienen un mayor poder de explicación. En términos globales, el coeficiente de determinación, la prueba conjunta $F$ y la prueba FRE presentaron mejores resultados en los modelos logarítmicos. De la misma manera, las variables construidas presentan estimadores positivos y significativos acordes con lo que se buscaba.

Ahora bien, los resultados por entidad mostraron a Baja California como un estado mayormente manufacturero que concentra su producción en dos municipios, destacando Tijuana, el municipio más grande tanto en la entidad como en la muestra. Sin embargo, en términos per cápita no está lo suficientemente dotado de infraestructura como cabría suponer.

El crecimiento de la población en Tijuana es elevado -concentra la mitad de la población del estado-. La capacidad de dotación de infraestructura del gobierno se ve limitada por dicho crecimiento. No obstante, presenta resultados positivos y significativos en IPE. Por su parte, Mexicali, cuya población crece en índices menores al promedio nacional, presenta niveles elevados en el IGP.

Sonora es un estado más balanceado en lo que respecta a los tres municipios de la muestra. Siendo Hermosillo el que mejores IGP presenta, así como el IPE. Nogales, a pesar de contar con sólo 7\% de la población y tener una tasa de crecimiento poblacional similar a la de Tijuana, no se encuentra en la desventaja que ahí se presenta y manifiesta resultados positivos y significativos en las estimaciones. 
Chihuahua se encuentra en el mismo caso que Baja California. La disparidad en la dotación de infraestructura entre Ciudad Juárez y la capital no concuerda con la participación de Ciudad Juárez en la economía de la entidad. Ciudad Juárez es el segundo municipio en tamaño de población y en nivel de producción dentro de la muestra y en las estimaciones tiene resultados positivos, pero no significativos.

Piedras Negras, el municipio fronterizo de Coahuila, presenta la mayor dotación de infraestructura de toda la muestra. Los coeficientes son elevados, positivos y significativos. Junto con Nogales, es uno de los municipios con mayor dotación de infraestructura y que presentan mayores coeficientes asociados al ingreso per cápita. Saltillo tiene resultados positivos pero no significativos, mientras que en Torreón los estimadores son significativos y positivos.

Los municipios de Tamaulipas cuentan con una equilibrada dotación de infraestructura entre ellos. Sin embargo, es la entidad con los índices más bajos de la muestra, por lo tanto, el resultado de las estimaciones es negativo en la mayor parte de los casos a excepción de Matamoros. Únicamente en Reynosa los datos son significativos; es decir, debido a la poca dotación de infraestructura en el estado, no se puede decir que representen un factor de crecimiento en dicha entidad. Los coeficientes negativos se pueden interpretar de dos maneras: ya sea que la falta de infraestructura les impide crecer o que pierden ingreso en comparación de los municipios mejor equipados.

Existe una relación positiva entre los municipios con más infraestructura con estimadores positivos y significativos, tal es el caso de Piedras Negras, Nogales y Torreón, en los estados de Coahuila y Sonora. En Chihuahua y Baja California, debido a su tamaño, crecen más rápido de lo que sus gobiernos pueden dotarlos con infraestructura. Sin embargo, su relación es positiva. Mexicali y Chihuahua (capital), a pesar de tener mayor dotación de infraestructura, presentan relaciones positivas pero no significativas debido al efecto que Tijuana y Juárez pueden inducir en relación con la concentración de la producción manufacturera en esas localidades.

\section{Conclusiones}

Existe evidencia de que la dotación de infraestructura -específicamente la infraestructura productiva- afecta positivamente en el crecimiento econó- 
mico de los municipios de la frontera norte con una elasticidad de 0.3875 con efectos fijos en los interceptos para el caso del modelo logarítmico y un coeficiente de 1.0813 para el modelo lineal.

Los resultados encontrados confirman efectivamente que la infraestructura en energía, transporte y telecomunicaciones son elementos determinantes del crecimiento de las ciudades fronterizas. Sin embargo, la infraestructura en transportes no se presenta como factor determinante, lo que puede deberse a que, al tratarse de estados fronterizos, en realidad no requieren de amplias redes carreteras al interior del país, sino más bien al exterior, lo cual hace más fluido el acceso al mercado mundial de bienes y servicios.

Una segunda causa podría deberse a las limitaciones del indicador que no toma en cuenta la fluidez con que pueden desplazarse por esas carreteras; y finalmente podría deberse a efectos de la información, como ya se ha advertido en otros estudios (Noriega y Fontela, 2007). La infraestructura en energía es la más importante de las tres ya que beneficia directamente a la industria manufacturera de exportación. Las comunicaciones benefician en su mayoría a los individuos sobre las industrias.

Para el caso de la infraestructura social, el número de escuelas no resulta significativo cuando se estima por sí solo ni cuando se estima con el IPE. Las capitales de los estados concentran a la población estudiantil, mientras que los municipios fronterizos, a pesar de contar con mayor población, tienen un menor número de escuelas. Una posible causa de lo poco significativo que resultó ser el número de escuelas es que la industria manufacturera concentra la mayor parte de la población económica. Esta industria se caracteriza por ser mayormente intensiva en mano de obra, por lo que suele no requerir mano de obra especializada, desviando así a los estudiantes hacia el trabajo o las actividades laborales.

\section{Bibliografía}

Aschauer, D. (1990), "Why is infrastructure important?", Federal Reserve Bank of Boston Conference Series, 21-68.

Barro, R. (1990), "Government spending in a simple model of endogeneous growth", The Journal of Political Economy, vol. 98, núm. 5, parte 2.

Becerril Torres, O., I. Álvarez Ayuso, L. E. Moral Barrera y R. Vergara González, (2009), "Indicador de infraestructuras productivas por entidad federativa en México, 1970-2003”, Gestión y Política Pública, vol. XVIII, núm. 2, pp. 379-438. 
Buhr, W. (2009), "Infrastructure of the Market Economy", Econ, Volkswirtschaftliche Diskussionsbeiträge, núm. 132-09, pp. 1-74.

Buitrago, R. (2009), "Reformas comerciales (apertura) en América Latina: revisando sus impactos en el crecimiento y el desarrollo", Revista Facultad de Ciencias Económicas: Investigación y Reflexión, vol. xvII, núm. 2, pp. 119-131.

Calderón Villarreal, C. (2006), "Apertura económica, salarios y migración internacional”, Análisis Económico, vol. XXI, núm. 46, pp. 167-187.

Cancelo de la Torre, J. R. y P. Uriz Tomé (1994), "Una metodología general para la elaboración de índices complejos de dotación de infraestructuras”, Estudios Regionales, núm. 40, pp. 167-188.

Capello, R. (2006), "La economía regional tras cincuenta años: desarrollos teóricos recientes y desafíos futuros”, Investigaciones regionales, núm. 9, pp. 169-192.

Castro, D. (2007), "Disparidad salarial urbana en México, 1992-2002”, Estudios Sociales, vol. xv, núm. 29, pp. 117-154.

Chiquiar, D. (2005), "Why Mexico's regional income convergence broke down", Journal of Development Economics, núm. 77, pp. 264-266.

Cobacho Tornel, M. B. y M. Bosch Mossi (2005), "Contrastes de hipótesis en datos de panel", XIII Jornadas de ASEPUMA, 2-7.

Dall'erba, S. (2007), Distribution of regional income and regional funds in Europe 19891999: an exploratory spatial data analysis, obtenido de Munich Personal RePEc Archive <http://mpra.ub.uni-muenchen.de/1378/>.

Dautrey, P. (2008), "Desarrollo y apertura económica en México: la eterna cuestión de los eslabones perdidos", Revista de Investigaciones Políticas y Sociológicas, vol. 7, núm. 2, pp. 153-166.

De Haan, J., W. Romp y J. E. Sturm (2007), "Public Capital and Economic Growth: Key Issues for Europe", International seminar on strengthening public investment and managing fiscal risks from public-private partnerships, Budapest, Hungría.

De Hoyos, R. y N. Lustig (2009), "Apertura comercial, desigualdad y pobreza", El Trimestre Económico, vol. LXxvı, núm. 302, pp. 283-328.

De la Fuente, A. (2008), "Inversión en infraestructuras, crecimiento y convergencia regional”, Fundación de Estudios de Economía Aplicada, Colección Estudios Económicos, pp. 1-14.

Díaz-Bautista, A. (2003), "El TLCAN y el crecimiento económico de la frontera norte de México", Comercio Exterior, vol. 53, núm. 12, pp. 1090-1097.

Domar, E. (1946), "Capital expansion, rate of growth, and employment”, Econométrica, vol. 14, núm. 2, pp. 140-145.

Feltenstein, A. y J. Ha (1995), "The role of infrastructure in mexican economic reform”, The World Bank Economic Review, vol. 9, núm. 2, pp. 287-304.

Fuentes, N. A. (2003), "Crecimiento económico y desigualdades regionales en México: el impacto de la infraestructura”, Región y Sociedad, vol. xv, núm. 27, pp. 81-103. 
Fuentes, N. A. (2007), "Las disparidades municipales en México: un estudio desde la óptica de la desigualdad”, Problemas del Desarrollo, vol. 38, núm. 150, julioseptiembre, pp. 232-233.

Fuentes, N. A. y C. Fuentes (2003), “Apertura comercial y divergencia económica regional en México; una propuesta de financiamiento de infraestructuras públicas", en N. Fuentes Flores, Crecimiento con convergencia o divergencia en las regiones de México, asimetría centro-periferia, México, El Colegio de la Frontera Norte, Plaza y Valdés.

Fuentes, N. A. y E. Mendoza (2003a), "Convergencia e infraestructura", en N. A.-B.P. Fuentes Flores, N. Fuentes Flores, Crecimiento con convergencia o divergencia en las regiones de México, asimetría centro-periferia, México, El Colegio de la Frontera Norte, Plaza y Valdés.

Fuentes, N. A. y E. Mendoza (2003b), "Infraestructura pública y convergencia regional en México, 1980-1998”, Comercio Exterior, vol. 53, núm. 2, febrero, pp. 178-187.

Fujita, M. y P. Krugman (2004), "The new economic geography: past, present and the future”, Regional Science 83, 139-149, 158-162.

Gerber, J. (2003), “Are incomes converging along the US-Mexico border?”, conferencia presentada en El Colegio de la Frontera Norte.

Germán-Soto, V. (2005), “(Di)convergencia regional en México”, Noesis, vol. 15, núm. 27, pp. 17-44.

Greene, W. (2002), Econometric analysis, Estados Unidos, Prentice Hall.

Harrod, R. (1939), "An essay in dinamic theory", The Economic Journal, vol. 49, núm. 193 , pp. 1-24.

Heckscher, E. (1991), "The effect of foreign trade on the distribution of income", en H. Flam y J. Flanders, Heckscher-Ohlin Trade Theory, Inglaterra, MIт Press.

Hicks, J. (1963), Una aportación a la teoría del ciclo económico, España, Aguilar.

Kaldor, N. (1963), Ensayo sobre desarrollo económico, México, Centro de Estudios Monetarios Latinoamericanos.

Keynes, J. (1992), Teoría general de la ocupación, el interés y el dinero, México, Fondo de Cultura Económica.

Lächler, U. y D. Aschauer (1998), "Public investment and economic growth in Mexico”, The World Bank. Policy Research Working Paper, núm. 1964, pp. 17-18.

Malthus, T. (1986), Ensayo sobre el principio de la población, México, Fondo de Cultura Económica.

Mayorga, M. y E. Muñoz (2000), "La técnica de datos de panel”, Banco Central de Costa Rica 8.

Mendoza, J. E. y A. Díaz-Bautista (2006), Economía regional moderna. Teoría y práctica, México, El Colegio de la Frontera Norte, Universidad de Guadalajara, Plaza y Valdés. 
Nijkamp, P. y B. Ubbels (1999), "Infrastructure, suprastructure and ecostructure: a portfolio of sustainable growth potentials", Faculteit der Economische Wetenschappen en Econometric, Research Memorandum 1999-51, pp. 1-18.

Noriega, A. y M. Fontenla (2007), "La infraestructura y el crecimiento económico en México”, El Trimestre Económico, vol. Lxxiv(4), núm. 296, pp. 885-900.

Ocegueda Hernández, J. M. (2007), “Apertura comercial y crecimiento económico en las regiones de México", Investigación Económica, vol. LXVI, núm. 262, pp. 89137.

Ohlin, B. (1971), Comercio interregional e internacional, España, Oikos.

Ricardo, D. (1973), Principios de economía política y tributación, España, Ayuso.

Richardson, H. (1986), Economía regional y urbana, México, Alianza Universidad.

Robinson, J. (1974), Ensayos de economía poskeynesiana, México, Fondo de Cultura Económica.

Robinson, J. (1973), Ensayos sobre la teoría del crecimiento económico, México, Fondo de Cultura Económica.

Romer, P. (1994), “The origins of endogenous growth", The Journal of Economic Perspectives, vol. 8 , núm. 1.

Ros, J. (2008), "La desaceleración del crecimiento económico en México desde 1982”, El Trimestre Económico, vol. Lxxv, núm. 299, pp. 537-560.

Sala-i-Martin, X. (1996), "Regional cohesion: evidence and theories of regional growth and convergence”, European Economic Review 40, pp. 1325-1352.

(1995), "The classical approach to convergence analisis", Economic Working Paper 117, pp. 1-43.

Smith, A. (1958), Investigación sobre la naturaleza y causas de la riqueza de las naciones, México, Fondo de Cultura Económica.

Solow, R. (1956), "A contribution to the theory of economic growth", The Quarterly Journal of Economics, vol. 70, núm. 1, pp. 91-94.

Urbano, P. M. (2005), "El papel de las infraestructuras públicas en el desarrollo regional", Noesis, vol. 15, núm. 17, pp. 45-67.

Wooldridge, J. M. (2002), Econometric analysis of cross section and panel data, Estados Unidos, MIT Press.

Artículo recibido el 26 de enero de 2011 Segunda versión recibida el 14 de diciembre de 2011

Artículo aprobado el 12 de enero de 2012 


\section{Anexo}

\section{Cuadro A. Variables utilizadas}

\begin{tabular}{|c|c|c|}
\hline Variable & Periodo & Fuente \\
\hline Ingreso estatal & 1993-2005 & $\begin{array}{l}\text { INEGI; Banco de Información } \\
\text { Económica; Cuentas } \\
\text { nacionales; Producto Interno } \\
\text { Bruto por entidad federativa }\end{array}$ \\
\hline Ingreso municipal & 1993, 1998, 2003 & $\begin{array}{l}\text { INEGI; Censos Económicos } \\
\text { 1994, 1999, } 2004 .\end{array}$ \\
\hline Población municipal & 1990, 1995, 200, 2005 & $\begin{array}{l}\text { INEGI; XI Censo General de } \\
\text { Población y Vivienda 1990; } \\
\text { I Conteo de Población y } \\
\text { Vivienda 1995; XII Censo } \\
\text { General de Población y } \\
\text { Vivienda 2000; II Conteo de } \\
\text { Población y Vivienda } 2005 .\end{array}$ \\
\hline Valor total de los activos fijos & 1993, 1998, 2003 & $\begin{array}{l}\text { INEGI; Censos Económicos } \\
\text { 1994, 1999, } 2004 .\end{array}$ \\
\hline Producción bruta total & 1993, 1998, 2003 & $\begin{array}{l}\text { INEGI; Censos Económicos } \\
\text { 1994, 1999, } 2004 .\end{array}$ \\
\hline Personal ocupado total & 1993, 1998, 2003 & $\begin{array}{l}\text { INEGI; Censos Económicos } \\
\text { 1994, 1999, } 2004 .\end{array}$ \\
\hline Valor agregado censal bruto & 1993, 1998, 2003 & $\begin{array}{l}\text { INEGI; Censos Económicos } \\
\text { 1994, 1999, } 2004 .\end{array}$ \\
\hline $\begin{array}{l}\text { Fuentes de abastecimiento } \\
\text { de agua }\end{array}$ & 1993-2005 & INEGI; \\
\hline $\begin{array}{l}\text { Anuario estadístico del estado } \\
\text { de Baja California }\end{array}$ & $\begin{array}{l}\text { 1994, 1995, 1996, 1997, 1998, } \\
\text { 1999, 2000, 2001, 2002, 2003, } \\
\text { 2004, 2005, 2006; }\end{array}$ & \\
\hline $\begin{array}{l}\text { Anuario estadístico del estado } \\
\text { de Sonora }\end{array}$ & $\begin{array}{l}\text { 1994, 1995, 1996, 1997, 1998, } \\
\text { 1999, 2000, 2001, 2002, 2003, } \\
\text { 2004, 2005, 2006; }\end{array}$ & \\
\hline $\begin{array}{l}\text { Anuario estadístico del estado } \\
\text { de Chihuahua }\end{array}$ & $\begin{array}{l}\text { 1994, 1995, 1996, 1997, 1998, } \\
\text { 1999, 2000, 2001, 2002, 2003, } \\
2004,2005,2006,\end{array}$ & \\
\hline
\end{tabular}




\begin{tabular}{|l|l|l|}
\hline \multicolumn{1}{|c|}{ Variable } & \multicolumn{1}{|c|}{ Periodo } & \\
\hline Anuario estadístico del estado & $\begin{array}{l}1994,1995,1996,1997,1998, \\
1999,2000,2001,2002,2003, \\
\text { de Coahuila }\end{array}$ & \\
\hline Anuario estadístico del estado & $\begin{array}{l}1994,1995,1996,1997,1998, \\
1999,2000,2001,2002,2003, \\
\text { de Tamaulipas }\end{array}$ & \\
\hline Tomas eléctricas domiciliarias & $1993-2005$ & \\
\hline Transformadores & $1993-2005$ & \\
\hline de distribución & $1993-2005$ & \\
\hline Longitud de líneas & $1993-2005$ & \\
\hline Carreteras principales & $1993-2005$ & \\
\hline Carreteras secundarias & $1993-2005$ & \\
\hline Carreteras rurales & $1993-2005$ & \\
\hline Camiones de carga & $1993-2005$ & \\
\hline Aeropuertos & $1993-2005$ & \\
\hline Vuelos comerciales & $1993-2005$ & \\
\hline Oficinas de telégrafos & $1993-2005$ & \\
\hline Oficinas postales & $1993-2005$ & \\
\hline Televisión por cable & $1993-2005$ & \\
\hline Escuela elemental & $1993-2005$ & \\
\hline Escuela primaria & $1993-2005$ & \\
\hline Capacitación para el trabajo & $1993-2005$ & \\
\hline Escuela secundaria & $1993-2005$ & \\
\hline Escuela profesional media & $1993-2005$ & \\
\hline Escuela medio superior & & \\
\hline & & \\
\hline
\end{tabular}

